\title{
BACKGROUND PAPER:
}

\section{FUNCTIONS OF CORONAVIRUS GLYCOPROTEINS}

\author{
Kathryn V. Holmes and Richard K. Williams \\ Department of Pathology \\ Uniformed Services University of the Health Sciences \\ Bethesda \\ Maryland 20814 \\ USA
}

For coronaviruses, the glycoproteins have both historical and biological significance $(1,2)$. Because some coronaviruses were difficult to isolate and propagate, their characteristic large, petal-shaped spikes first allowed coronaviruses to be identified as related agents in a common family. Viral glycoproteins play important roles throughout the life cycle of coronaviruses. Viral glycoproteins interact with receptors on the host cell membrane and are required for penetration of the viral genome into cells by fusion of the viral envelope with the plasma membrane or endosomal membranes. In virus-infected cells, coronavirus glycoproteins may be glycosylated, acylated, oligomerized, cleaved into subunits by proteases, and transported to specific membrane compartments. The viral glycoproteins participate in assembly of virions, and the intracellular location of viral glycoproteins may determine the location of coronavirus budding. Viral glycoproteins on the surface of infected cells may permit fusion with adjacent cells, and may make the cell susceptible to immune cytolysis or cell-mediated cytotoxicity, or permit hemadsorption. Epitopes of viral glycoproteins may be recognized by neutralizing, non-neutralizing or hemagglutination-inhibiting antibodies. Thus, elucidation of the structure and functions of coronavirus glycoproteins is of central importance in understanding the replicative cycle and pathogenesis of coronaviruses.

A model of a coronavirus virion is shown in Figure 1. All coronaviruses contain the membrane glycoprotein, (M), and the large spike glycoprotein, (S). Virions of serologic groups II and IV may also contain the third glycoprotein, the hemagglutinin esterase (HE), which forms a smaller spike in the viral envelope. These include bovine coronavirus (BCV), human coronavirus OC43 (HCV-OC43), some strains of mouse hepatitis virus (MHV), hemagglutinating encephalomyelitis virus of swine (HEV), and turkey coronavirus (TCV). The amount of $\mathrm{HE}$ relative to $S$ in virions may vary considerably.

\section{BIOCHEMICAL CHARACTERISTICS OF CORONAVIRUS ENVELOPE GLYCOPROTEINS}

The membrane glycoprotein, (M), has a molecular weight in different coronaviruses which ranges from 20 to $30 \mathrm{~K}$. A small portion of the amino terminus extends from the outer surface of the lipid bilayer. On some coronaviruses, eg. MHV, this domain is glycosylated by addition in the Golgi apparatus of short oligosaccharides to serine or threonine residues. In IBV and some other coronaviruses, the oligosaccharides of $\mathrm{M}$ are $\mathrm{N}$-linked. The membrane glycoprotein spans the membrane 3 times, and has a large domain beneath the lipid bilayer.

The glycoprotein which makes up the large spike, S, has an apparent molecular weight of 150 to $200 \mathrm{~K}$ and appears to be a dimer held together by non-covalent bonds. A small carboxy- 


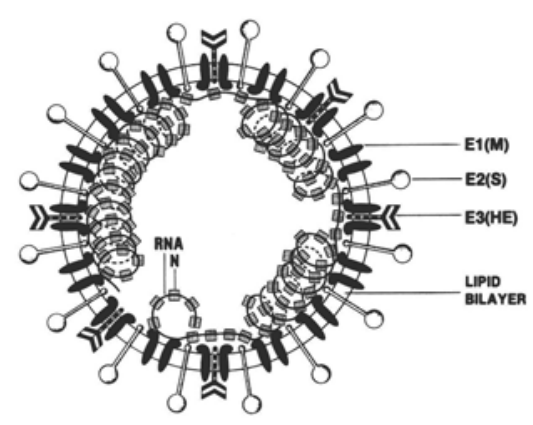

Figure 1. Model of coronavirus virions. The envelopes of all coronaviruses contain membrane glycoprotein, (M) and the spike, (S) glycoprotein. Hemagglutinating coronaviruses contain, in addition, a third glycoprotein hemagglutinin esterase (HE) which forms a short spike on their envelopes. A helical nucleocapsid composed of the nucleocapsid protein $\mathrm{N}$ and the plusstrand, genomic RNA is enclosed within the viral envelope. (Reproduced with permission from Academic Press.)

terminal domain rich in cysteine residues is located beneath the lipid bilayer, and the protein traverses the lipid bilayer only once. During maturation and intracellular transport, some molecules of $S$ are cleaved by host cell proteases probably located in the Golgi apparatus to yield two large subunits called S1 and S2. The cleavage is host-cell dependent. Palmitic acid is covalently linked to the carboxy-terminal subunit of $S 2$ which is anchored in the lipid bilayer. Numerous N-linked oligosaccharides are found on both of the subunits of this glycoprotein. Aggregation may occur at alkaline $\mathrm{pH}$.

The 65K HE glycoprotein forms dimers linked together by disulfide bonds which have an apparent molecular weight of $140 \mathrm{~K}$. Each of the glycoproteins of several coronaviruses has been cloned, sequenced and expressed in eukaryotic cells.

\section{FUNCTIONS OF CORONAVIRUS ENVELOPE GLYCOPROTEINS}

Table 1 summarizes our current understanding of the functions of the three coronavirus glycoproteins. The membrane glycoprotein M of MHV interacts with the nucleocapsid in vitro and may aid in virus budding within infected cells by interaction of the cytoplasmic domain with the viral nucleocapsid. Because budding commences in the Golgi apparatus where this glycoprotein accumulates, it is possible that the intracellular location of $\mathrm{M}$ determines the site of virus maturation.

The large spike glycoprotein $\mathrm{S}$ has two functions which are essential for coronavirus infectivity. For MHV-A59, S interacts with a $110 \mathrm{~K}$ glycoprotein receptor on the surface of susceptible murine cells, leading to infection. Blocking of the virus-binding domain of this receptor by monoclonal antireceptor antibody prevents infection of the cells. Comparable glycoprotein receptors for other coronaviruses have not yet been identified. S glycoproteins of many coronaviruses cause virus-induced cell fusion. For MHV, protease cleavage may occur either intracellularly, during glycoprotein synthesis and transport through the Golgi apparatus, or extracellularly, using trypsin. The S glycoproteins in IBV, MHV and BCV virions have protease-susceptible sites, but there is no protease susceptible site in the $S$ glycoprotein of FIPV, although this virus can cause cell fusion. Different host cells vary in their susceptibility to fusion with coronaviruses. It is likely but not proven that the cell fusing activity of coronaviruses is caused by the same glycoprotein that fuses the viral envelope with host cell membranes during virus penetration. For IBV and TGEV, $S$ is responsible for hemagglutination. The biological significance of this activity and the characteristics of the receptor for $S$ on erythrocytes are not yet understood. The $S$ glycoprotein stimulates both neutralizing and fusion-inhibiting antibodies. Neutralizing monoclonal antibodies directed against $S$ have been used to select virus variants which show altered pathogenicity in vivo. The domains of $S$ which are responsible for its biological activities are now being analyzed. 
Table 1. Functions of coronavirus envelope glycoproteins

M

Interaction with nucleocapsid

Virion assembly

Determination of site virus budding

$S$

Binding to $110 \mathrm{~K}$ receptor glycoprotein (MHV)

Cell fusion (protease activated for MHV and BCV, not FIPV)

Stimulation of neutralizing antibody

Stimulation of fusion-inhibiting antibody

Hemagglutination (TGEV, IBV)

HE

Hemagglutination (BCV, OC43, HEV, TCV)

Binding to 9-O-acetylated neuraminic acid (BCV, OC43)

Acetylesterase (BCV, OC43)

Hemagglutination by BCV, OC43, HEV, TCV and some strains of MHV is apparently due to interaction of the $\mathrm{HE}$ glycoprotein with a carbohydrate moiety on the surface of erythrocytes. The HE of BCV and HCV-OC43 recognize 9-O-acetylated sialic acid, the same binding moiety used by the influenza $\mathrm{C}$ glycoprotein. In addition, these glycoproteins have an acetylesterase activity which can cleave off the 9-O-acetyl group, thereby preventing hemagglutination. A central question is whether infection of cells in vitro or in vivo can result from the recognition of 9-O-acetylated sialic acid by the $\mathrm{HE}$ glycoprotein. If $\mathrm{HE}$ can function as a viral attachment protein leading to infection, then it will be important to determine whether S or HE is responsible for fusion of the viral envelope with cellular membranes. The effects upon infection of monoclonal antibodies directed against HE may help to elucidate the role of this glycoprotein in coronavirus infection. For MHV-JHM, there is a suggestion that the HE glycoprotein of this murine virus may be important for infection of the central nervous system of rats. A virus which expresses the mRNA which should encode the HE glycoprotein was selected by passage of MHV-JHM through rat brain. If the HE activity is not required for growth of the virus in cell culture, then virus which has lost the capacity to make HE could outgrow virus which expresses HE.

It is fascinating to consider how serogroups II and IV of the coronavirus family acquired the gene encoding the $\mathrm{HE}$ glycoprotein, which resembles in part the glycoprotein of influenza $\mathrm{C}$. This additional glycoprotein may provide these coronaviruses with a broader host range and/or altered pathogenicity in comparison with coronaviruses which encode only the $M$ and $S$ glycoproteins. A careful study of the receptor recognition mechanisms of many coronaviruses may provide new insight into the evolution of the coronavirus family.

\section{ACKNOWLEDGEMENTS}

The authors are grateful for discussions with Dr. Lawrence S. Sturman and Dr. Mark F. Frana. This research was supported by NIH grant \#18997. Dr. Williams is supported by NRSA \#F32AI07810. The opinions or assertion contained herein are the private views of the authors and should not be construed as official or necessarily reflecting the views of the Uniformed Services University of the Health Sciences or the Department of Defense.

\section{REFERENCES}

1. Holmes, K.V. Replication of Coronaviruses, in Virology, 2nd Edition, B.N. Fields et al.., (eds) Raven Press, New York, in press, 1989.

2. Spaan, W., Cavanagh, D., Horzinek, M.C., Coronaviruses: Structure and genome expression. J. Gen. Virol. 69: 2939-2952, 1988. 